\title{
Síndrome anti-GQ1b: Descripción de cuatro pacientes y revisión de la literatura
}

\author{
REINALDO URIBE S.M., FELIPE SUÁREZ H.a,
} PATRICIO SANDOVAL R., PATRICIO MELLADO T.

\section{Anti-GQ1b syndrome. Report of four cases}

Anti-GQ1b syndrome includes Miller Fisher Syndrome (MFS), Guillain Barré Syndrome (GBS), Bickerstaff s brain stem encephalitis (BBE) and Acute Ophtamoplegia (AO). We report four patients aged 16 to 76 years, with anti-GQ1b syndrome. All presented with MFS, one of them evolved to GBS pharyngeal-cervical-brachial variant and other to GBS with BBE. All had a previous history of diarrhea or upper respiratory tract infection. All had positive anti-GQ1b serum antibodies. Both brain magnetic resonance imaging and cerebrospinal fluid analysis were normal. Electrophysiology studies were compatible with a demyelinating disease. Two patients needed airway protection with an orotracheal tube and developed dysautonomia. All four patients were treated with immunomodulation. On the sixth month follow-up, patients had only minimal alterations in the neurological examination.

(Rev Med Chile 2013; 141: 1211-1215).

Key words: Encephalitis; Gangliosides; Guillain Barré syndrome; Miller Fisher syndrome; Ophthalmoplegia.

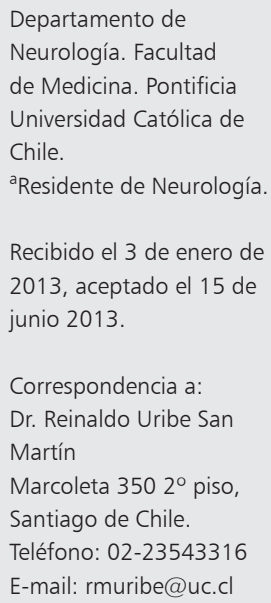

$\mathrm{D}$ urante la última década se ha abierto un amplio campo de investigación sobre la patogenia autoinmune de algunas neuropatías periféricas como el síndrome de Guillain Barré (SGB), la polineuropatía desmielinizante inflamatoria crónica, la neuropatía motora multifocal y la neuropatía paraproteinémica. Evidencia sustancial implica como base una respuesta inmune humoral frente a distintos tipos de gangliósidos ${ }^{1}$. En este sentido, el síndrome de Miller Fisher (SMF), caracterizado por la presencia de oftalmoparesia, ataxia y arreflexia se ha asociado a la presencia de anticuerpos anti-GQ1b, hallazgo extendido a otras enfermedades como la romboencefalitis de Bickerstaff (EB), la oftalmoparesia aguda (OA) y algunos SGB clásicos ${ }^{2}$. No obstante, el papel exacto que desempeñan estos anticuerpos aún se desconoce. Nosotros presentamos cuatro casos con anticuerpos anti-GQ1b resumidos en la Tabla 1 y 2.

\section{Casos}

\section{Paciente 1}

Hombre de 16 años con antecedentes de infección respiratoria y diarrea autolimitadas una semana previo al ingreso, tres días después comenzó con disartria, diplopía, disfagia, parestesias en extremidades y ataxia. Al examen tenía disartria, oftalmoparesia de rectos superiores, inferiores y laterales, biparesia facial, paresia velopalatina, cefaloparesia, reflejos osteotendineos (ROT) abolidos, dismetría bilateral y ataxia. Se estudió con resonancia magnética (RM) de encéfalo que resultó normal, electromiografía (EMG) que mostró polineuropatía desmielinizante generalizada, sin disociación albúmino citológica en líquido cefalorraquídeo (LCR) y anticuerpos anti-GQ1b positivos en suero. Evolucionó con paresia de extremidades superiores y requirió intubación orotraqueal para manejo de vía aérea. Se estableció el 
Tabla 1. Resumen de los hallazgos clínicos de los pacientes

\begin{tabular}{|c|c|c|c|c|}
\hline & Paciente 1 & Paciente 2 & Paciente 3 & Paciente 4 \\
\hline Diagnóstico: & SMF/SGB & SMF/SGB/EB & SMF & SMF \\
\hline Edad: & 16 años & 46 años & 42 años & 76 años \\
\hline Sexo: & Masculino & Masculino & Femenino & Masculino \\
\hline $\begin{array}{l}\text { Antecedentes: } \\
\text { - } \quad \text { Diarrea } \\
\text { - } \quad \text { IR Alta } \\
\text { - } \quad \text { Tiempo del antec. }\end{array}$ & $\begin{array}{l}\text { Sí } \\
\text { Sí } \\
7 \text { días }\end{array}$ & $\begin{array}{l}- \\
7 \text { días }\end{array}$ & $\begin{array}{l}\text { Sí } \\
- \\
2 \text { días }\end{array}$ & $\begin{array}{l}\text { Sí } \\
\text { Sí } \\
10 \text { días }\end{array}$ \\
\hline \begin{tabular}{ll}
\multicolumn{2}{l}{ Síntomas: } \\
- & Tiempo síntomas \\
- & Disartria \\
- & Diplopía \\
- & Ptosis \\
- & Vértigo \\
- & Disfagia \\
- & Parestesias \\
- & Alt. Marcha
\end{tabular} & $\begin{array}{l}4 \text { días } \\
\text { Sí } \\
\text { Sí } \\
- \\
- \\
\text { Sí } \\
\text { Sí } \\
\text { Sí }\end{array}$ & $\begin{array}{l}2 \text { días } \\
\text { Sí } \\
\text { Sí } \\
- \\
\text { Sí } \\
- \\
- \\
\text { Sí }\end{array}$ & $\begin{array}{l}1 \text { día } \\
- \\
\text { Sí } \\
- \\
- \\
- \\
- \\
-\end{array}$ & $\begin{array}{l}7 \text { días } \\
\text { Sí } \\
- \\
\text { Sí } \\
- \\
\text { Sí } \\
- \\
\text { Sí }\end{array}$ \\
\hline $\begin{array}{ll}\text { Examen neurológico: } \\
\text { - } & \text { Disartria } \\
\text { - } & \text { Midriasis } \\
\text { - } & \text { RFM disminuido } \\
\text { - } & \text { OCM } \\
\text { - } & \text { Nistagmus } \\
\text { - } & \text { PFP } \\
\text { - } & \text { Paresia velo } \\
\text { - } & \text { Cefaloparesia } \\
\text { - } & \text { ROT } \\
\text { - } & \text { Dismetría } \\
\text { - } & \text { Disdiadococinesia } \\
\text { - } & \text { Ataxia } \\
\text { - } & \text { Romberg }\end{array}$ & $\begin{array}{l}\text { Sí } \\
- \\
- \\
\text { 2RS, 2RI y 2RL } \\
- \\
\text { Bilateral > der } \\
\text { Sí } \\
\text { Sí } \\
\text { Abolidos } \\
\text { Sí } \\
- \\
\text { Sí } \\
-\end{array}$ & $\begin{array}{l}\text { Sí } \\
\text { Sí } \\
\text { Sí } \\
\text { RSI } \\
\text { Sí } \\
- \\
- \\
- \\
\text { Abolidos } \\
\text { Sí } \\
- \\
- \\
\text { Sí }\end{array}$ & $\begin{array}{l}- \\
- \\
- \\
2 R L \\
- \\
- \\
- \\
- \\
\text { Aumentados } \\
- \\
- \\
- \\
-\end{array}$ & $\begin{array}{l}\text { Sí } \\
\text { Sí } \\
\text { Sí } \\
\text { Oftalmoplejia } \\
- \\
\text { Bilateral > der } \\
\text { Sí } \\
- \\
\text { Abolidos } \\
\text { Sí } \\
\text { Sí } \\
\text { Sí } \\
-\end{array}$ \\
\hline
\end{tabular}

En la Tabla se describen las distintas características de los pacientes. - : No. SMF: S. Miller Fisher, SGB: S. Guillain Barré, EB: Romboencefalitis de Bickerstaff, IR: infección respiratoria, antec: antecedente, alt: alteración, RFM: reflejo fotomotor, OCM: oculomotilidad, PFP: parálisis facial periférica, ROT: reflejos osteotendíneos, RS: músculo recto superior, RI: músculo recto inferior, RL: músculo recto lateral, RSI: músculo recto superior izquierdo, EESS: extremidades superiores, Der: derecha.

diagnóstico de SMF que evolucionó a SGB variante faringo cérvico braquial. Se inició tratamiento con inmunoglobulina intravenosa (IgIV), pero desarrolló shock anafiláctico aunque tenía niveles de inmunoglobulina A normales. Se continuó tratamiento con plasmaféresis hasta completar 15 volemias. En control a los 6 meses, se pesquisó mínima biparesia facial y ROT disminuidos.

\section{Paciente 2}

Hombre de 46 años con antecedentes de infección respiratoria la semana previa, luego de cinco días comenzó con disartria, diplopía, vértigo y ataxia. $\mathrm{Al}$ examen se objetivó disartria, midriasis con reflejos fotomotores (RFM) disminuidos, paresia del recto superior izquierdo, nistagmus bihorizontal, ROT abolidos, dismetría bilateral y Romberg positivo. Se estudió con RM encéfalo normal. La EMG presentó alteración del reflejo de pestañeo. Sin disociación en LCR y anticuerpos anti-GQ1b positivos en suero. Evolucionó con sopor, midriasis arrefléctica, biparesia facial y paresia de extremidades superiores. Requirió intubación para manejo de vía aérea y monitorización por disautonomía. Se establecieron los diagnósticos de SMF, SGB y EB. Recibió IgIV (0,4gr/kg/día por 
Síndrome anti-GQ1b - R. Uribe et al

Tabla 2. Resumen de los exámenes y evolución de los pacientes

\begin{tabular}{|c|c|c|c|c|}
\hline & Paciente 1 & Paciente 2 & Paciente 3 & Paciente 4 \\
\hline Diagnóstico: & SMF/SGB & $\mathrm{SMF} / \mathrm{SGB} / \mathrm{EB}$ & SMF & SMF \\
\hline $\begin{array}{l}\text { Estudio: } \\
-\quad \text { Ac. anti-GQ1b } \\
-\quad \text { Disociación A-C } \\
-\quad \text { EMG } \\
\text { - } \quad \text { RM encéfalo }\end{array}$ & $\begin{array}{l}\text { Positivo } \\
\text { - } \\
\text { Desmielinizante } \\
\text { Generalizado } \\
\text { Normal }\end{array}$ & $\begin{array}{l}\text { Positivo } \\
\text { - } \\
\text { Alteración } \\
\text { R. pestañeo } \\
\text { Normal }\end{array}$ & $\begin{array}{l}\text { Positivo } \\
\text { - } \\
\text { Desmielinizante } \\
\text { facial } \\
\text { Normal }\end{array}$ & $\begin{array}{l}\text { Positivo } \\
- \\
\text { Desmielinizante } \\
\text { Generalizado } \\
\text { Normal }\end{array}$ \\
\hline $\begin{array}{l}\text { Evolución hospitalaria: } \\
\text { - Nuevos signos }\end{array}$ & Paresia EESS & $\begin{array}{l}\text { Sopor, midriasis, PFP } \\
\text { Oftalmoplejia } \\
\text { Paresia EESS, ataxia }\end{array}$ & $\begin{array}{l}\text { Abolición ROT } \\
\text { Ataxia }\end{array}$ & Leve cefaloparesia \\
\hline $\begin{array}{ll}\text { - } & \text { Disautonomía } \\
\text { - } & \text { Intubación } \\
\text { - } & \text { Días hospitalización }\end{array}$ & $\begin{array}{l}- \\
\text { Sí, sin VM } \\
18\end{array}$ & $\begin{array}{l}\text { Sí } \\
\text { Sí, sin VM } \\
20\end{array}$ & $\begin{array}{l}- \\
- \\
10\end{array}$ & $\begin{array}{l}\text { Sí } \\
- \\
20\end{array}$ \\
\hline $\begin{array}{l}\text { Tratamiento: } \\
\text { - } \quad \text { IgIV } \\
\text { _ } \quad \text { Plasmaféresis }\end{array}$ & $\begin{array}{l}\text { Sí } \\
\text { Shock anafiláctico } \\
\text { Sí }\end{array}$ & Sí & $\begin{array}{l}\text { Sí } \\
\text { - }\end{array}$ & $\begin{array}{l}\text { Sí } \\
-\end{array}$ \\
\hline $\begin{array}{l}\text { Evolución ambulatoria: } \\
-\quad \text { Meses } \\
\text { - } \quad \text { Ex. Neurológico }\end{array}$ & $\begin{array}{l}6 \\
\text { Biparesia facial } \\
\text { ROT disminuidos }\end{array}$ & $\begin{array}{l}6 \\
\text { Normal }\end{array}$ & $\begin{array}{l}6 \\
\text { Mínimo RL derecho }\end{array}$ & $\begin{array}{l}6 \\
\text { Midriasis, 2RL } \\
\text { ROT disminuidos, } \\
\text { mínima ataxia }\end{array}$ \\
\hline
\end{tabular}

En la Tabla se describen las distintas características de los pacientes. -: No. SMF: S. Miller Fisher, SGB: S. Guillain Barré, EB: Romboencefalitis de Bickerstaff, PFP: parálisis facial periférica, ROT: reflejos osteotendíneos, Ac: anticuerpos, A-C: albúmino citológica, EMG: electromiografía, RM: resonancia magnética, IgIV: inmunoglobulina intravenosa, RL: músculo recto lateral, EESS: extremidades superiores, Der: derecha y VM: ventilación mecánica.

5 días consecutivos). A los 6 meses tenía examen neurológico normal.

\section{Paciente 3}

Mujer 42 años sana, presentó dos días previos al ingreso diarrea y diplopía. Se pesquisó paresia de rectos laterales y ROT exaltados. Se estudió con RM encéfalo normal, la EMG mostró polineuropatía desmielinizante en zona facial, sin disociación en LCR y anticuerpos anti-GQ1b positivos en suero. Evolucionó con abolición de ROT y ataxia. Se estableció el diagnóstico de SMF. Recibió IgIV en dosis habitual. A los 6 meses presentaba mínima paresia del recto lateral derecho.

\section{Paciente 4}

Hombre de 76 años sano, diez días previos presentó una infección respiratoria y diarrea, a los tres días comenzó con disartria, ptosis, disfagia y ataxia. $\mathrm{Al}$ examen tenía disartria, midriasis con
RFM disminuidos, oftalmoplejia bilateral, biparesia facial, paresia velopalatina, ROT abolidos, dismetría y ataxia. Se estudió con RM encéfalo normal, EMG con polineuropatía desmielinizante generalizada, sin disociación en LCR y anticuerpos anti-GQ1b positivos en suero. Evolucionó con cefaloparesia y disautonomía. El diagnóstico fue SMF. Recibió IgIV y en su examen al $6^{\circ}$ mes presentaba midriasis bilateral, oftalmoparesia leve de rectos laterales y ROT disminuidos.

\section{Discusión}

Describimos cuatro pacientes con anticuerpos anti-GQ1b en suero, todos con características de SMF. Descrito en 1956 por Charles Miller Fisher en tres pacientes con la triada oftalmoplejia, ataxia y arreflexia. En su artículo, un paciente presentó parálisis facial y debilidad, similar a dos 
de nuestros casos. Fisher concluyó que esta forma de polineuropatía correspondería a una variante del SGB, pensamiento que se anticipó a las futuras descripciones que correlacionan inmunológicamente ambos cuadros basados en la presencia de anticuerpos anti-GQ1b ${ }^{3}$.

En 1957, Bickerstaff describe ocho pacientes con romboencefalitis manifestada por compromiso de conciencia, alteración oculomotora y ataxia, hallazgos similares al SMF${ }^{4}$. Por otra parte, en 1996 Yuki y cols. separan la oftalmoplejia aguda sin ataxia, describiendo ocho casos con oftalmoparesia, ROT conservados, sin ataxia y con anticuerpos anti-GQ1b ${ }^{5}$. Trabajos posteriores de Yuki, Odaka e Hirata, intentando aclarar la relación nosológica entre estas patologías, describen 194 pacientes con anticuerpos anti-GQ1b en el suero. Encontraron al igual que en nuestros casos, una clara sobreposición en las manifestaciones clínicas, las cuales conformarían un continuo, basado en un mecanismo autoinmune común, acuñando el término "síndrome de anticuerpos anti-GQ1b"2.

Estudios en humanos con anticuerpos contra el gangliósido GQ1b, detectan importantes realces en uniones neuromusculares y husos de músculos oculomotores $^{2,6}$. Por otra parte, existe una conexión epidemiológica entre la infección por Haemophilus influenzae, Mycoplasma pneumoniae y Campylobacter jejuni con la posterior aparición de la sintomatología oculomotora. Esta asociación se basa en la similitud entre gangliósidos bacterianos y los de axones motores, fenómeno llamado mimetismo molecular ${ }^{2,7}$. Sin embargo, esta asociación es heterogénea y no absoluta, encontrándose casos de otros tipos de enfermedades, con altos títulos de anti-GQ1b que nunca cursan con oftalmoplejia ${ }^{5,8}$ y que la presencia de anti-GQ1b es alrededor de $26 \%$ para SGB, $66 \%$ en la EB y $95 \%$ en $\mathrm{SMF}^{6}$.

Epidemiológicamente, de las series de pacientes con SGB, en especial dos estudios italianos sobre sus variantes regionales, refieren que entorno al 3 a 25\% de los casos corresponden al SMF $6,9,10$. La incidencia anual de este síndrome es de 0,1/100.000 habitantes, predominando su aparición en primavera $^{6,11}$.

Respecto a las manifestaciones clínicas, nuestros hallazgos son similares a los de Odaka y cols. ${ }^{2}$ :

- La oftalmoparesia: La presentan todos los pacientes con OA, SMF asociado a SGB, EB aislada o asociada al SGB. Incluye varias anomalías oftalmológicas como oftalmoplejia in- ternuclear, espasmos o fallas de convergencia y retracción de párpados ${ }^{6}$. Además, $50 \%$ presenta ptosis, alteraciones pupilares o de otros nervios craneanos $^{11}$.

- Hiporreflexia: Presente en todos los pacientes con SMF, SMF asociado a SGB o SGB aislado. Además, se observa en $91 \%$ de los pacientes con EB asociada a SGB, $67 \%$ de las EB aisladas y en $53 \%$ de las $\mathrm{OA}^{2}$.

- Ataxia: Observada en todos los SMF y EB. Además, en $68 \%$ de los pacientes con SMF asociado a SGB y $45 \%$ de las EB asociadas a $\mathrm{SGB}^{2}$.

El antecedente de infección previa se encontró en todos nuestros pacientes y se describe en general entre 60 y $80 \%$ de los casos ${ }^{2}$. La minoría llega a falla respiratoria y necesidad de ventilación mecánica, sin embargo, puede requerirse intubación para protección de vía aérea como sucedió en dos de nuestros pacientes, dado el compromiso de nervios craneanos bajos. Otras complicaciones graves son: coma, miocardiopatía por disautonomía, acidosis láctica y dolor ${ }^{6}$.

La disociación albúmino citológica se describe entre el 25 y $75 \%$ de los casos y aumenta a $84 \%$ en la $3^{\text {a }}$ semana de evolución ${ }^{12}$. Nosotros no encontramos este hallazgo seguramente por lo precoz del estudio, realizado entre el $1^{\text {er }}$ y $7^{\circ}$ día de iniciado los síntomas.

Aunque la mayoría son condiciones agudas y autolimitadas, existen varios reportes de recurrencia asociada a la presencia de anti-GQ1b ya sea en el SMF, EB o OA, incluso recurriendo hasta en siete ocasiones, pero siempre con evolución favorable ${ }^{13-15}$.

No encontramos alteraciones en las neuroimágenes y no existe un hallazgo imagenológico específico, pero en pacientes con SMF y principalmente en EB, la RM puede tener compromiso del troncoencéfalo y del núcleo del tercer nervio craneano en secuencias $\mathrm{T} 2^{6}$. También puede existir compromiso del tracto espinocerebeloso y pedúnculos cerebelosos. Lesiones periféricas se han reportado en raíces lumbosacras, cauda equina, columna posterior, ganglio sensitivo dorsal, tercer y cuarto nervio craneano ${ }^{6}$.

Los estudios electrofisiológicos muestran compromiso del sistema nervioso central por lentitud difusa en el electroencefalograma, ausencia del N20 cortical con preservación del N13 cervical y ausencia de R2 en el reflejo de pestañeo ${ }^{16,17}$. Por 
otra parte, el compromiso del sistema nervioso periférico se correlaciona con la presencia de arreflexia, mostrando alteraciones tipo desmielinizante $^{6}$. También se ha demostrado por electromiografía de fibra única, defectos presinápticos en la transmisión neuromuscular hasta tres meses después. Este hallazgo está estrechamente relacionado con la presencia de anticuerpos anti-GQ1b en las uniones neuromusculares ${ }^{18}$.

Sobre el tratamiento, aunque se ha preconizado el uso de corticoides, inmunoglobulina y plasmaféresis, no existen estudios que demuestren su eficacia ${ }^{19,20}$. Algunos investigadores han demostrado que el uso de inmunomoduladores, como anticuerpos monoclonales, recupera las anormalidades electrofisiológicas ${ }^{19}$. En estricto rigor, hay que considerar el curso evolutivo natural favorable que presenta la mayoría, siendo posible que varios no requieran tratamiento. No obstante, es complejo no ofrecer tratamiento a pacientes que evolucionan con compromiso neurológico grave, como los que fueron presentados y haciendo una extrapolación de los tratamientos demostrados en el SGB, utilizamos IgIV o plasmaféresis ${ }^{19,20}$.

\section{Referencias}

1. Ueda M, Kusunoki S. Autoimmune neuropathies: diagnosis, treatment, and recent topics. Brain Nerve 2011; 63: 549-55.

2. Odaka M, Yuki N, Hirata K. Anti-GQ1b IgG antibody syndrome: clinical and immunological range. J Neurol Neurosurg Psychiatry 2001; 70: 50-5.

3. Fisher M. An unusual variant of acute idiopathic polyneuritis (syndrome of ophthalmoplegia, ataxia and areflexia). N Engl J Med 1956; 255: 57-65.

4. Bickerstaff ER. Brainstem encephalitis: further observations on a grave syndrome with benign prognosis. $\mathrm{Br}$ Med J 1957; 1: 1384-7.

5. Yuki N. Acute paresis of extraocular muscles associated with IgG anti-GQ1b antibody. Ann Neurol 1996; 39: 668-72.

6. Lo Y. Clinical and inmunological spectrum of the MillerFisher syndrome. Muscle Nerve 2007; 36: 615-27.

7. Yuki N, Taki T, Takahashi M, Saito K, Yoshino H, Tai T, Handa S, Miyatake T. Molecular mimicry between GQ1b ganglioside and lipopolysaccharides of Campylobacter jejuni isolated from patients with Fisher's syndrome. Ann Neurol 1994; 36: 791-3.

8. Más-Lázaro C, García-Pastor A, Díaz-Insa S, MoltóJordà JM, Lacruz-Ballester L. Variante faringocervicobraquial del síndrome de Guillain-Barré: una entidad clínica bien definida con un perfil inmunológico heterogéneo. Rev Neurol 2008; 47: 579-81.

9. Govoni V, Granieri E, Tola MR, Casetta I, Ruppi P, Vaghi L. The frequency of clinical variants of Guillain-Barré in Ferrara, Italy. J Neurol 1999; 246: 1010-4.

10. Guillain-Barré syndrome variants in Emilia-Romagna, Italy, 1992-3: incidence, clinical features, and prognosis. Emilia-Romagna Study Group on Clinical and Epidemiological Problems in Neurology. J Neurol Neurosurg Psychiatry 1998; 65: 218-24.

11. Schabet M. Miller Fisher syndrome. Pract Neurol 2009; 9: 289-91.

12. Van Door P, Ruts L, Jacobs B. Clinical features, pathogenesis, and treatment of Guillian-Barré syndrome. Lancet Neurol 2008; 7: 939-50.

13. Orr C F, Storey C E. Recurrent Miller-Fisher syndrome. J Clin Neurosci 2004; 11: 307-9.

14. Sánchez V, Guerrero R, Verdugo R, Cartier L. Oftalmoplejia aguda sin ataxia, de carácter recurrente. Rev Chil Neuro-Psiquiatr 2010; 48: 147-51.

15. Heckmann JG, Dütsch M. Recurrent Miller Fisher syndrome: clinical and laboratory features. Eur J Neurol 2011. doi: 10.1111/j.1468-1331.2011.03584.x. [in press].

16. Ogawara K, Kuwabara S, Yuki N. Fisher syndrome or Bickerstaff brainstem encephalitis? Anti-GQ1b IgG antibody syndrome involving both the peripheral and central nervous systems. Muscle Nerve 2002; 26: 845-9.

17. Calleja J, García A, de Pablos C, Polo JM. Síndrome de Miller Fisher: estudio electrofisiológico evolutivo en cinco pacientes. Rev Neurol 1998; 27: 60-4.

18. Lo YL, Leoh TH, Dan YF, Lim LL, Seah A, Fook-Chong S, Ratnagopal P. Presynaptic neuromuscular transmission defect in the Miller Fisher syndrome. Neurology 2006; 66: 148-9.

19. Lo YL. Immunotherapy for anti-GQ1b IgG antibodymediated disorders: role of electrophysiology in human trials. Brain 2009; 132: 1-2.

20. Overell J, Hsieh S, Odaka M, Yuki N, Willison H. Treatment for Fisher syndrome, Bickerstaff's brainstem encephalitis and related disorders. Cochrane Database Syst Rev. 2007: CD004761. 